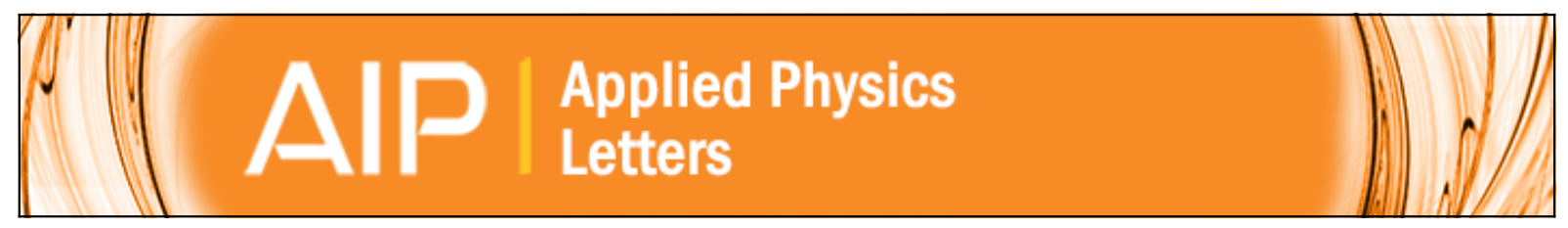

\title{
Optical properties of tetrapod-shaped CdTe nanocrystals
}

Davide Tarì, Milena De Giorgi, Fabio Della Sala, Luigi Carbone, Roman Krahne, Liberato Manna, Roberto

Cingolani, Stefan Kudera, and Wolfgang J. Parak

Citation: Applied Physics Letters 87, 224101 (2005); doi: 10.1063/1.2130727

View online: http://dx.doi.org/10.1063/1.2130727

View Table of Contents: http://scitation.aip.org/content/aip/journal/apl/87/22?ver=pdfcov

Published by the AIP Publishing

\section{Articles you may be interested in}

Study of the radiative recombination processes in tetrapodshaped CdTe nanocrystals

AIP Conf. Proc. 1199, 307 (2010); 10.1063/1.3295424

Growth kinetics of CdTe colloidal nanocrystals

J. Chem. Phys. 131, 084712 (2009); 10.1063/1.3212964

Radiative recombination dynamics in tetrapod-shaped CdTe nanocrystals: Evidence for a photoinduced screening of the internal electric field

Appl. Phys. Lett. 92, 191905 (2008); 10.1063/1.2924306

Size Dependent Photomodulated Transmission Spectroscopy of CdTe Tetrapodshaped Nanocrystals AIP Conf. Proc. 893, 1063 (2007); 10.1063/1.2730264

Exciton transitions in tetrapod-shaped CdTe nanocrystals investigated by photomodulated transmittance spectroscopy

Appl. Phys. Lett. 89, 094104 (2006); 10.1063/1.2335801
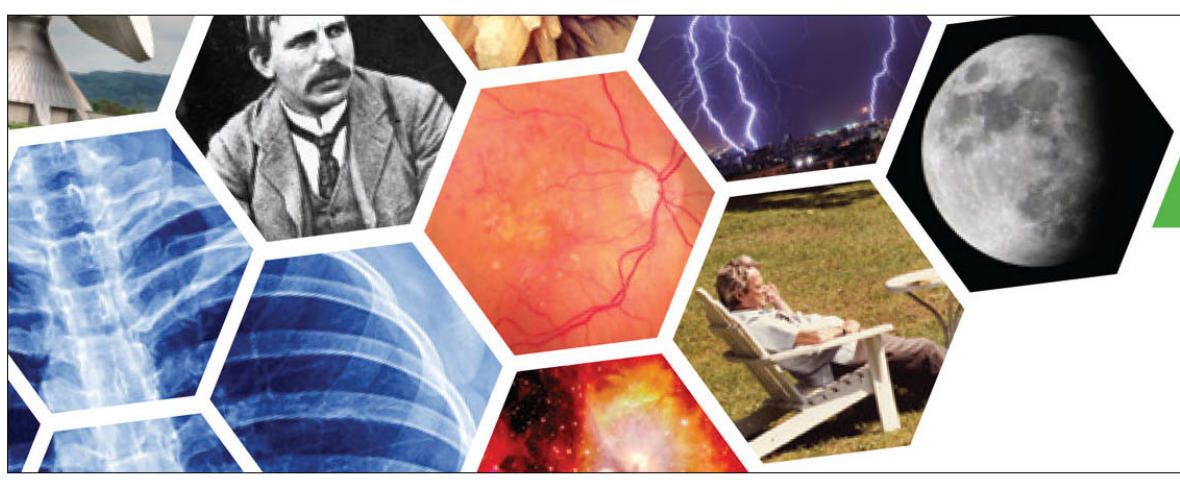

\section{SUBSCRIBE TO \\ physics today}




\title{
Optical properties of tetrapod-shaped CdTe nanocrystals
}

\author{
Davide Tarì, ${ }^{a)}$ Milena De Giorgi, Fabio Della Sala, Luigi Carbone, Roman Krahne, \\ Liberato Manna, and Roberto Cingolani \\ National Nanotechnology Laboratory of INFM clo Istituto Superiore Universitario di Formazione \\ Interdisciplinare, Università di Lecce, Via per Arnesano, 73100 Lecce, Italy
}

\author{
Stefan Kudera and Wolfgang J. Parak \\ Center for Nanoscience, Ludwig Maximilians Universität München, Munich, Germany
}

(Received 24 May 2005; accepted 19 September 2005; published online 21 November 2005)

\begin{abstract}
We studied the carrier confinement in tetrapod-shaped colloidal CdTe nanocrystals by means of absorption, photoluminescence, and photoluminescence excitation spectroscopy at room and cryogenic temperatures. The spectra show features characteristic of the tetrapod shape together with a clear dependence on the dominant confinement parameter, i.e., the diameter of the tetrapod arm. Theoretical calculations based on an envelope-function approximation and using the exact tetrapod shape have been performed to assign the observed spectral features. Oscillator strength and size dependence of the transitions energy have been calculated showing a direct correlation between the oscillator strength and the nanocrystal shape. (C) 2005 American Institute of Physics.
\end{abstract}

[DOI: $10.1063 / 1.2130727$ ]

In recent years, there has been a remarkable advancement in the ability to grow anisotropic nanocrystals, such as rods, ${ }^{1-3}$ tetrapods, ${ }^{4}$ and nanocrystals with even more complex branched shapes. ${ }^{5}$ The influence of the geometric shape on the electronic structure of the nanocrystals is of great interest, since it modifies their optical and electrical properties. Hu and co-workers, for instance, ${ }^{3}$ have shown that colloidal quantum rods exhibit linearly polarized light emission. Therefore, the opportunity to tune the nanocrystal shape paves the way to new properties stemming from the peculiar geometry of these structures. This has a great impact in different fields of nanotechnology, such as biological tagging, photovoltaics, electronics and light-emitting diodes (LEDs). An intriguing nanocrystal shape is the tetrapod [Fig. 1(a)], in which four rod-shaped arms branch with a wurtzite crystal structure from a central zinc blende core. ${ }^{4}$

In this letter, we study the electronic structure of CdTe tetrapods as a function of the length and diameter of the arms. Linear absorption spectra, photoluminescence excitation (PLE), and fluorescence experiments at room and low temperatures together with theoretical calculations were performed on samples of different geometrical dimensions. The tetrapods exhibit a remarkable fluorescence yield with well resolved multifold splitting of the spectra originating from the electron quantization in the arms of the nanostructure, as confirmed by theoretical calculations.

The tetrapod samples were grown according to the protocol described by Manna and co-workers. ${ }^{4}$ Figure 1(a) shows a schematic illustration of the tetrapod shape and sketches the band offset between the zinc blende core and the wurtzite arms. ${ }^{6}$ Structural information about tetrapod samples has been obtained by transmission electron measurements (TEM). In this letter, we discuss the properties of a set of tetrapods (labeled as $T 1, T 2$, and T3) whose geometrical dimensions were assessed by wide-field TEM images [Figs. 1(b)-1(d)). The tetrapod samples have a good homogeneity

\footnotetext{
a) Author to whom correspondence should be addressed; electronic mail
} davide.tari@unile.it in shape and a rather narrow distribution of arm diameters $(d)$ and lengths $(l)$, whose average values have been found to be equal to: $d=7 \mathrm{~nm}$ and $l=60 \mathrm{~nm}$ for the sample $T l, d$ $=5.7 \mathrm{~nm}$ and $l=30 \mathrm{~nm}$ for $T 2$ and finally, $d=4.7 \mathrm{~nm}$ and $l$ $=25 \mathrm{~nm}$ for $T 3$.

Figures 2(a)-2(c) shows the linear absorption and the fluorescence spectra of the three tetrapod samples recorded at room temperature in solution. In all absorption spectra, quantum confinement causes the lowest energy peak to blueshift with respect to the bulk CdTe value $(1.50 \mathrm{eV}$ at $T=300 \mathrm{~K})$, and a series of overlapping peaks appears at higher energies due to high-index exciton states. As a general trend, the absorption features of the tetrapods are size-dependent. In smaller tetrapods the lowest exciton state occurs at higher energy (1.81 eV for sample T3) compared with the larger tetrapods $(1.715 \mathrm{eV}$ for sample $T 1)$, and the energy spacing

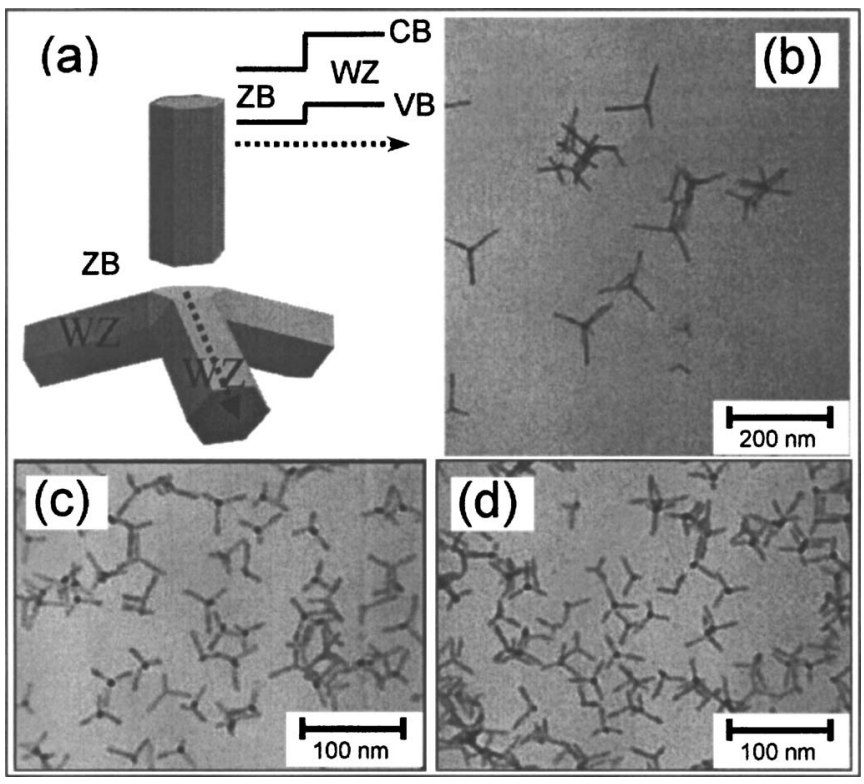

FIG. 1. (a) An illustration of a tetrapod. (b)-(d) TEM of three CdTe tetrapod samples. 


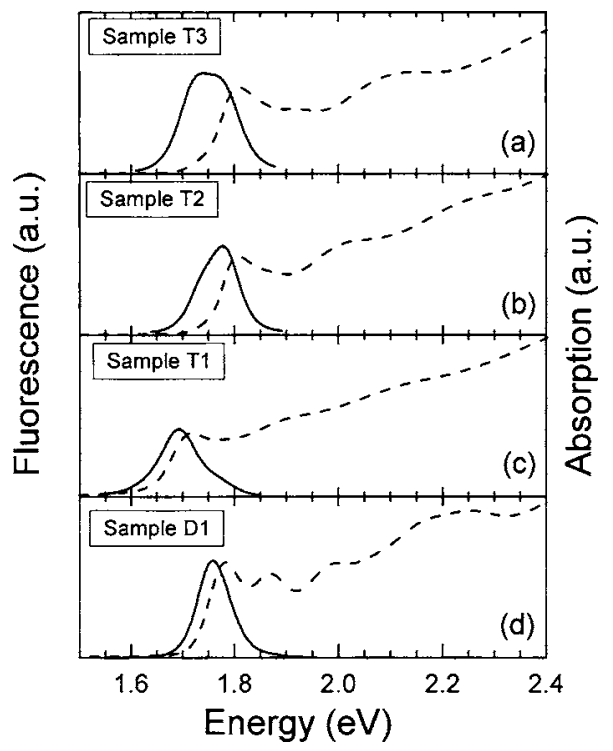

FIG. 2. Absorption (dashed line) and fluorescence spectra (solid line) taken at room temperature of (a)-(c) tetrapods and (d) dot samples.

between the various exciton states increases when the dimensions are reduced.

In order to understand the effect of the tetrapod shape on its overall electronic structure, it is useful to compare these nanostructures with nanospheres, widely studied in the last years. The absorption and emission spectra from spherical CdTe nanocrystals (sample D1), emitting in a comparable spectral range, are displayed in Fig. 2(d). The absorption spectra of the tetrapods are less distinct than in spheres, and this can be explained by the effect of the more elaborate geometry of the tetrapods on their electronic structure. Although the confinement in tetrapods depends more strongly on the diameter of the arms than on their length, the smaller degree of exciton confinement in the arms and their lower symmetry lead to a multitude of exciton states separated by comparatively smaller energy gaps.

By looking at the emission spectra from the tetrapods we clearly observe a double-peak structure in smaller tetrapods (samples $T 2$ and T3), whereas in larger tetrapods (sample T1) the second peak can be better resolved after a careful deconvolution of the spectrum. A detailed analysis of the emission spectra shows that a decrease in arm width of the tetrapods leads to an increase both in energy spacing between the two peaks and of the intensity of the high-energy peak. Moreover, in the tetrapod spectra displayed in Figs. 2(a)-2(c), the Stokes shift increases with decreasing size, in analogy with spherical nanocrystals, ${ }^{7}$ but it stays remarkably large (about $100 \mathrm{meV}$ for smaller tetrapods). For comparison, in spherical nanocrystals of diameter around $5 \mathrm{~nm}$, the Stokes shift is rather small (about $50 \mathrm{meV}$ ). The large Stokes shift observed in the tetrapods could partly result from the larger size distribution compared to dots ${ }^{8}$ and partly from the shape anisotropy, as observed in rods. ${ }^{3}$

Figures 3(a)-3(c) shows the room temperature PLE spectra of the three tetrapod samples. If the detection energy is tuned over the full spectral width of the fluorescence signal, no significant change in the characteristic features is observed. This proves that the double-peak structure in emission [resolved also in the PLE spectra, as indicated by the small arrows in Figs. 3(b) and 3(c)] originates from the same class of nanocrystals, i.e., the tetrapods. In the intermediate

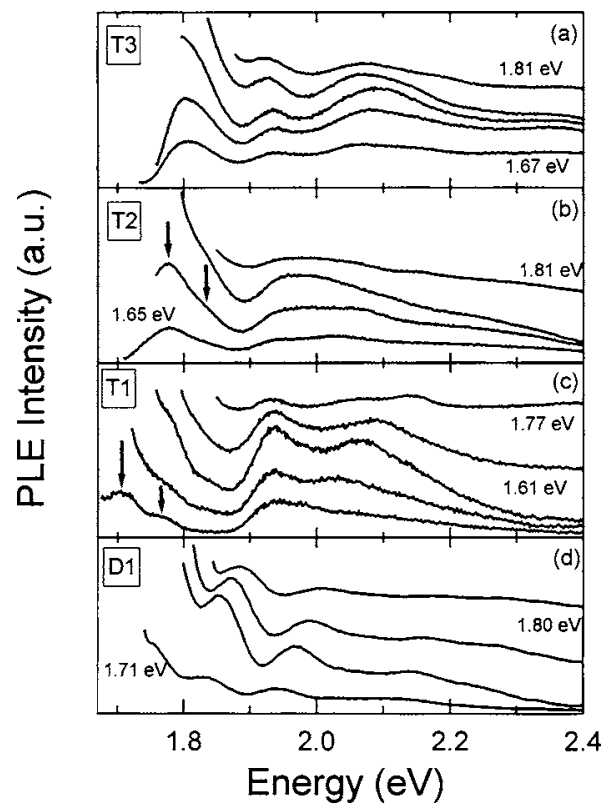

FIG. 3. Photoluminescence excitation spectra of (a)-(c) tetrapods and (d) dots at different detection energies for each sample. The small peak at $2 \mathrm{eV}$ that shifts with the detection energy is an artefact of the solvent.

detection energy range, the PLE signal results from two transitions, namely coming from the two peaks observed in fluorescence. This effect, together with the size distribution and the elaborate geometry of the tetrapods ${ }^{8}$ leads to an inhomogeneous broadening of the features, which prevents us from observing photoselection of nanocrystals with different sizes as normally observed in dot samples [like the one shown in Fig. 3(d)], where a blueshift of about $60 \mathrm{meV}$ of the higher energy states is observed when increasing the detection energy.

Measurements at cryogenic temperatures were performed by depositing a drop of nanocrystal solution on $\mathrm{SiO}_{2}$

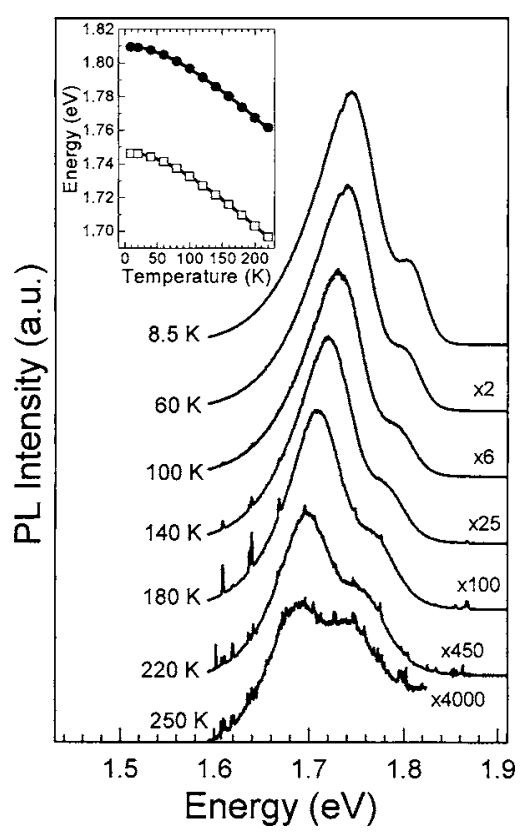

FIG. 4. Temperature-dependent PL spectra of the sample $T 2$. The different curves are shifted for clarity (corresponding magnification is indicated). The inset shows the peak positions fitted by the Varshni function for both transitions observed in the PL spectra. 


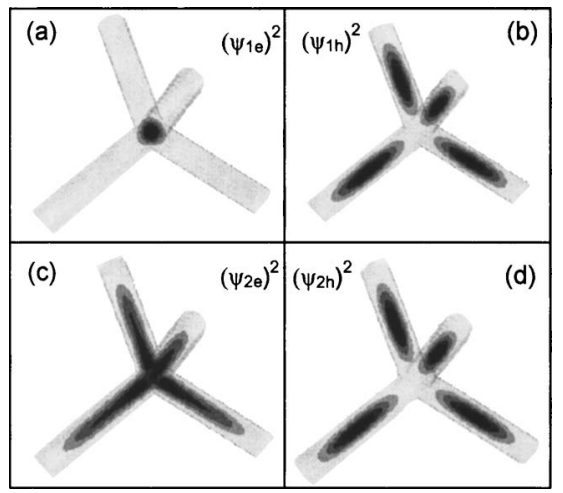

FIG. 5. Three-dimensional view of the isosurfaces of $\Psi^{2}$ of the electron (a) and hole (b) ground state and of the first electron (c) and hole (d) excited state calculated for the $T 2$ tetrapod.

substrates. Figure 4 shows PL spectra of the sample $T 2$ recorded in a temperature range between 8.5 and $250 \mathrm{~K}$. The ratio between the intensity of the high energy peak and the low energy peak varies significantly with increasing temperature, ${ }^{9}$ while the overall intensity of the signal decreases due to the increased rate of nonradiative processes. This behavior can be correlated with the thermal filling of the electronic levels due to a rise of the Fermi level at higher temperatures. Both peaks show a redshift with increasing temperature. In the inset of Fig. 4 we report the peak position of the two observed transitions as a function of the temperature. The experimental points for both transitions follow the Varshni equation ${ }^{10}$

$$
E_{g}(T)=E_{g}(0)-\frac{\alpha T^{2}}{\beta+T},
$$

where $E_{g}$ is the energy gap of material, $T$ is the temperature, and $\alpha$ and $\beta$ are Varshni's coefficients.

For a more complete understanding of the optical properties of the tetrapods, we performed preliminary theoretical calculations of the electronic structure, based on the envelope-function approximation, and using the geometrical dimensions (arm lengths and diameters) derived from the TEM measurements. We modeled the tetrapod shape, by considering a zinc blende (ZB) octahedron as the core and four wurtzite (WZ) hexagonal prisms as the arms and by solving numerically the three-dimensional single-particle effective-mass Schrödinger equations. ${ }^{11} \mathrm{We}$ also assumed that the electrons are confined by a potential barrier whose height is equal to the electron affinity of $\mathrm{CdTe}^{12}$ whereas the holes are confined by an infinite potential barrier. In the following, we discuss the results for the sample $T 2$.

For the $T 2$ sample, the calculated excitation energies for the ground (GS) and the first excited (EX) states (GS $=1.674 \mathrm{eV}$ and $\mathrm{EX}=1.747 \mathrm{eV}$, respectively) agree well with the PL experimental peak. The squared wave functions of the first two confined electron and hole states for the $T 2$ sample are shown in Fig. 5. We found that the ground state of the electrons has a $A_{1}$ symmetry, with the electron density mostly localized in the core of the tetrapod [Fig. 5(a)], whereas the wave function of the ground state of the holes has a $T_{2}$ symmetry and spreads into the arms [Fig. 5(b)]. On the other hand, both the electron and hole wave functions of the first excited state have $T_{2}$ symmetry, with the carriers completely localized in the arms [Figs. 5(c) and 5(d)]. As a result of the different electron-hole overlap, the oscillator strength for the transition between the ground state levels is much smaller (0.52) than that between the first excited states (1.76), i.e., the probability that the excited state recombines radiatively is increased. In addition, due to the different symmetry of the two electron states, the probability of an intraband transition which leads the carrier from the first excited state to the ground state is reduced. Thus the two peaks observed in the fluorescence spectra can be ascribed to the transition of the two lowest confined electron and hole states. The peak at lower energy should be correlated to the transition between the ground state in conduction band and to the ground state in valence band $\left(E_{l e} \rightarrow E_{l h}\right)$, whereas the peak at higher energy to the transition between the first excited states in conduction and valence band $\left(E_{2 e} \rightarrow E_{2 h}\right)$.

In conclusion, we investigated the photoluminescence properties of tetrapod-shaped nanocrystals as a function of their size. We observed that the absorption, PL, and PLE spectra show also features peculiar of their complex shape. A theoretical calculation which takes into account the exact shape of the tetrapods and based on the envelope-function approximation made possible to understand the origin of these features and to assign the observed experimental energy transitions.

The authors gratefully acknowledge the useful discussion from Dr. Marco Anni and P. Pompa for the PLE measurements. They also thank the expert technical help of P. Cazzato. This work has been partially supported by the SANANO European project Contract No. STRP013698, MIURFIRB project, the Deutscher Akademischer Austauschdienst (DAAD) and the Vigoni Foundation.

${ }^{1}$ X. G. Peng, L. Manna, W. D. Yang, J. Wickman, E. Scher, A. Kadavanich, and A. P. Alivisatos, Nature (London) 404, 59 (2000).

${ }^{2}$ T. Mokari and U. Banin, Chem. Mater. 15, 3955 (2003); C. Pacholski, A. Kornowski, and H. Weller, Angew. Chem., Int. Ed. 41, 1188 (2002).

${ }^{3}$ J. T. Hu, L. S. Li, W. D. Yang, L. Manna, L. Wang, and A. P. Alivisatos, Science 292, 2060 (2001).

${ }^{4}$ L. Manna, D. J. Milliron, A. Meisel, A. Scher, and A. P. Alivisatos, Nat. Mater. 2, 382 (2003).

${ }^{5}$ D. J. Milliron, S. M. Hughes, Y. Cui, L. Manna, J. Li, L. W. Wang, and A. P. Alivisatos, Nature (London) 430, 190 (2004); K. A. Dick, K. Deppert, M. W. Larsson, T. Martensson, W. Seifert, L. R. Wallenberg, and L. Samuelson, Nat. Mater. 3, 380 (2004).

${ }^{6}$ M. Nirmal, D. J. Norris, M. Kuno, and M. G. Bawendi, Phys. Rev. Lett. 753728 (1995); A. L. Efros, M. Rosen, M. Kuno, M. Nirmal, D. J. Norris, and M. Bawendi, Phys. Rev. B 54, 4843 (1996); Z. H. Yu, J. B. Li, D. B. O'Connor, L. W. Wang, and P. F. Barbara, J. Phys. Chem. B 107, 5670 (2003).

${ }^{7}$ D. J. Norris, A. L. Efros, M. Rosen, and M. Bawendi, Phys. Rev. B 53, 16347 (1996).

${ }^{8}$ The peak around $2 \mathrm{eV}$ has been attributed to the solvent by performing PLE measurements on the solution in the absence of nanocrystals.

${ }^{9} \mathrm{By}$ a rough fit of the ratio between the intensity of the two PL peaks as a function of the temperature and considering one Arrhenius plot we obtain an activation energy which falls within the $10 \%$ margin of error of the energy spacing between the two states, as measured from the PL spectra.

${ }^{10}$ J. Perez-Conde, A. K. Bhattacharjee, M. Chamarro, P Lavallard, V. D. Petrikov, and A. A. Lipoovskii, Phys. Rev. B 64, 113303 (2001).

${ }^{11}$ I. Calderon-Hernandez, edited by M. C. Tamargo (New York, 2001); F. Long, W. E. Hagston, P. Harrison, and T. Stirner, J. Appl. Phys. 82, 3414 (1997); Y. Yan, M. M. Al-Jassim, K. M. Jones, S. H. Wei, and S. B. Zang, Appl. Phys. Lett. 77, 1461 (2000); S. H. Wei and S. B. Zhang, Phys. Rev. B 62, 6944 (2000).

${ }^{12}$ I. Visoly-Fisher, A. Sitt, M. Wahab, and D. Cahen, ChemPhysChem 6, 277 (2005). 